\title{
Considerations in the Selection of Retainer Following an Orthodontic Treatment
}

\author{
Nikoleta Ivanova, DMD ${ }^{1}$, Nora Gogusheva, DMD $^{1}$, MiroslavaDinkova, DMD, PhD $^{1,2}$ \\ ${ }^{1}$ MMD Dental Center of Orthodontics, Sofia, Bulgaria \\ ${ }^{2}$ Associate Professor, Department of Orthodontics
}

Faculty of Dental Medicine, Medical University - Sofia, Bulgaria

\begin{abstract}
Retention is the phase of orthodontic treatment which maintains the teeth in their orthodontically corrected position. The right choice of retainer selection is essential forthe reduction of relapse risk after orthodontic treatment. The Aim of the study was to establish the considerations of retainer selection for reducing the frequency of relapse following an orthodontic treatment.Materials and Methods: The present study included 403 randomly selected patients, aged 7 to 61, treated and monitored by same specialist orthodontist, between 1999 and 2014. Patients were grouped by age (group 1 (6-12), group 2 (12-21), group 3 (21-40), group 4 (40-61)), type and severity of malocclusion, periodontal status, type of orthodontic appliances, period and type of retention based on data from their records, study models and photo documentation. Results: It was found a correlation between the age, type and severity of malocclusion, periodontal status, type of orthodontic appliances, cooperation of the patient and the choice of retainer. The most commonly used retainers in all patient groups were established. Conclusion: Orthodontic retainers counteract the natural tendency of teeth to return to their original position under the influence of periodontal, occlusal and soft tissue forces.
\end{abstract}

Keywords: Orthodontics, Retainer, Aligner, Trainer, Dent@lign

\section{Introduction}

Retention is the phase of orthodontic treatment which maintains the teeth in their orthodontically corrected position following the end of the active orthodontic treatment [1].Orthodontic retainers counteract the natural tendency of teeth to return to their original position under the influence of periodontal, occlusal and soft tissue forces [2].The duration of retention period corresponds to the time needed for recovery and stabilization of periodontal tissues. The right choice of retainer is essential to reduce the risk of relapse and to stabilize the teeth and periodontal tissues. The evaluation of retention phase importance demands a long-term controlled study for identifying the considerations for selection of retainer appliances.

Previous studies have shown that after completion of active orthodontic treatment supracrestal periodontal fibers remain extended over 7 months [3, 4]. This is the minimum time needed for periodontal fibers to remodel to the new position of the teeth [3]. According to research conducted in England, the most common retention period lasts about 12 months [5]. For each patient the duration of the retention phase varies depending on the individual factors. For example, in patients with a history of severe periodontal disease, diastemas and/or spacing [6], permanent retention is recommended. Longer retention is necessary in patients with a history of previous root resorption or bone loss. In such patients there is evidence of increased risk of crowding of the lower incisors after the retention period [7]. Such retention is needed in patients with a change more than 2 $\mathrm{mm}$ in the front-back position of the lower incisors, i.e. the degree of inclination.

The retainer appliances are classified as removable (RR)also as fixed retainers (FR). Both groups are divided into several subgroups. For example Hawley,Essixretainersand vacuum retainers are removable. Lingual retainers from cuspid to cuspid (3-3), cuspid and cuspid (wire is connected only with the two canines, incisors remain free), fixed retainer from premolar to premolar (4-4), double fixed retainer (DFR)are referred to the group of fixed retainers. Fixed retainers can be made of wire with different thickness and diameter, as well as from composite fibers. The wire can be round orthodontic, flexible, spiral or multi-threaded.

Kerim was the first to report on the use of fixed retainers [8]. In 1977 Zachrisson published the advantages of multithreaded wire to earlier round orthodontic wire used forelaboration of fixed retainers [9]. Multi-threaded wire increases the mechanical retention of the composite without the need of retention curves and due to its flexibility allows the physiological movement of the teeth, although fixed adjacent teeth [10]. Later, in 1982,Zachrisson and Artun offered a technique that involves attaching the wire only to canines [11]. In 1983, Zachrisson proposes fixing the wire to all teeth in the front segment [11].

\section{Aim}

The aim of this study was to establish the considerations of retainer selectionfor reducing the frequency of relapse following an orthodontic treatment.

\section{Materials and Methods}

The present study included 403 randomly selected patients aged from 7 to 61 years of age. All the patients were treated and monitored by the same specialist-orthodontist, between 1999 and 2014. Patients were grouped by age (Group 1 (612), Group 2 (12-21), Group 3 (21-40), Group 4 (40-61)), type of malocclusion (crowding, diastema, overjet, deep overbite, open bite, Class II Angle relationship, Class III Angle relationship, cross bite) and severity of malocclusion

Volume 6 Issue 1, January 2017 


\section{International Journal of Science and Research (IJSR) \\ ISSN (Online): 2319-7064}

Index Copernicus Value (2015): 78.96 | Impact Factor (2015): 6.391

(mild, moderate, severe, need for extractions), periodontal status (history of gingivitis (mild, moderate, severe), periodontal disease (mild, moderate, severe)), type of the orthodontic appliances (fixed or removable appliances, extraoral appliances, myofunctional appliances and aligners), period of retention ( $<12$ months, 12-48 months, $>$ 48 months), type of the retainer (fixed FR, vacuum retainers (VR), plates, aligners, myofunctional appliances and combination of the above), based on patients records, study models andphoto documentation. Tha data was entered and processed with statistical package SPSS 13.0.1. For level of significance, which rejects the null hypothesis, was chosen $\mathrm{p}<0.05$.

\section{Results}

\section{Age and choice of retainer}

From statistical processing a correlation between the age of the patient and the choice of retainer was established. In the first age group the most commonly used retainer was the lingual plate (45.2\%), in Group 2 - FR from canine to canine (3-3)in 53\%, in Group 3 - FR (3-3) in 44.3\%, followed by relatively higher percentage of a combination of FR and VR (18.6\%); in Group 4 with the highest percentage was the of double fixed retainer (35.4\%), followed by a combination of FR and VR (26\%). (Table 1).

Table 1: Distribution of used retainers to the age groups

\begin{tabular}{|c|c|c|c|c|c|c|c|c|}
\hline $\begin{array}{r}\text { Type of } \\
\text { retainer } \\
(\%)\end{array}$ & $\begin{array}{c}\text { FR } \\
(3-3)\end{array}$ & $\begin{array}{c}\text { Ling } \\
\text { ual } \\
\text { plate }\end{array}$ & VR & $\begin{array}{c}\text { Combi } \\
\text { nation } \\
\text { ofFR } \\
(3-3)+ \\
\text { VR }\end{array}$ & DFR & FR & FR \\
$(4-4)$ & $(2-2)$ & Other \\
Age group & & & & & & \\
\hline Group 1 & 7 & 56.1 & 24.2 & - & - & 2.3 & 6.1 & 4.3 \\
\hline Group 2 & 53 & 8.9 & 18 & 4.4 & - & 9.1 & 4 & 2.6 \\
\hline Group 3 & 44.3 & - & 5.3 & 18.6 & 7 & 17.5 & 4.2 & 3.1 \\
\hline Group 4 & 18 & - & 4.2 & 26 & 35.4 & 11.4 & 1.2 & 3.8 \\
\hline
\end{tabular}
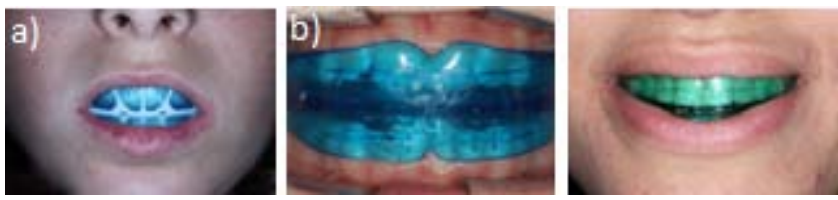

Type and severity of malocclusion and choice of retainer.The evaluation of type and severity of malocclusion and the type of retainer showed that in extraction cases FR from premolar to premolar was placed in a relatively large percentage (63\%), in severe crowding cases - FR (43.2\%). After treatment of diastemas, caused by bad habits (sucking habits, reverse swallow and other), the combination of FR and VR was the most common (68.1\%). In cases with deep bite a FR in the lower jaw in a combination with Trainer for night use were usedfor retention in $59.5 \%$ of the cases. (Fig.2c))The treatment of bruxism/bruxomania in 93.2\% ended with TMJ appliance. In the presence of impacted wisdom teeth and contraindications for their extraction and/or non-cooperative patient in $71.4 \%$ was applied a combination of FR and VR, with control visits and stripping on every 6 months. Treatment of open bite with a risk of relapse ended with VR and attachments for intermaxillary traction in $38.2 \%$.

\section{Periodontal status and selection of retainer.}

The patients studied were grouped according to the history of periodontal disease - gingivitis and periodontal disease (mild, moderate and severe). In patients with a history of mild to moderate gingivitis and mild periodontal disease with the greatest use were FR (48.3\%), followed by VR (26.3\%). In moderate gingivitis and advanced stages of periodontal diseasea greater use of DFR (39.7\%) and FR + VR (29.2\%) wasseen. (Fig. 1)
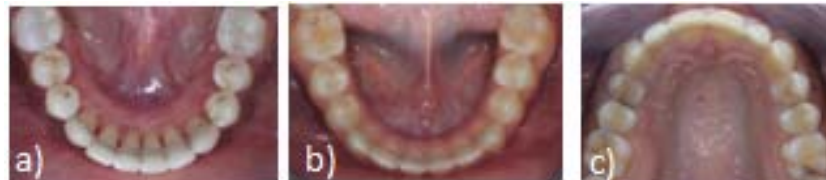

Figure 1: (a) Patient after periodontal and orthodontic treatment. DFR was made aiming to stabilize the periodontal tissues. (b) FRfrom canine to canine in lower jaw. (c) FRfrom 12 to 22 in the upper jaw.

Type of orthodontic appliances and selection retainer. According to the type of orthodontic appliances and the type of used retainer,the patients were distributed in the following way: during treatment with lingual plate, most often the last one was remained for retention (64.9\%); in treatment with fixed appliances - FR (80.4\%); when treated with EOA (extra oral appliances)- a combination of FR + VR (54.9\%); after treatment with aligners - the last one remained for retention in $73.7 \%$ of cases, as well as in cases treated withTrainer system - the last Trainer was used for retention (89.5\%). (Fig. 2a) and 2b))

Fig.2(a) Patient after myofunctional orthodontic teratment. Myobrace retention (Myofunctional Research Co.) (b) Trainer for Kids c) Adult patient with Trainer for Alignment for retention.

Treated jaw and choice of retainer.

From the conducted study it was found that the retainer with the highest frequency in the upper jaw was FR (3-3) - 57\%, followed by FR (2-2) - 18\% and VR - 15\%. In lower jaw FR (3-3) was also the most common used method for result retention after conducted orthodontic treatment - 64\%, followed by FR + VR $-11.3 \%$ and FR from premolar to premolar $-9.6 \%$.

\section{Cooperation of the patient and choice of retainer.}

A statistically significant correlation between the patient manifested cooperativeness and the used retainer was established. In $89.9 \%$ of patients, which were irregular in their treatment appointments and had a history of lost appliances and/or lost/broken brackets a FR were used. The cooperative patients treated with removable appliances were indicated for a removable retention in $67.4 \%$ of cases.

\section{Discussion}

Several studies in the literature are focused on comparison of fixed and removable retainers. The current research established that for the choice of the retainer appliances there are certain considerations. 


\section{International Journal of Science and Research (IJSR) \\ ISSN (Online): 2319-7064}

Index Copernicus Value (2015): 78.96 | Impact Factor (2015): 6.391

Age and choice of retainer.In the current study in elderly patients the most common used retainer was the fixed retainer, which can be associated with employment and social life of the patient, i.e. with the need for aesthetic and casual retainer system, providing 24 hour retention.

Literature research shows that due to the higher average age of patients in the group of fixed retainers, clinicians are more willing to put fixed retainers to adult patients [11, 12 , 13]. There is also a complicated periodontal pathology in elderly patients. Relieving the load on supporting tissues and providing periodontal health requires a fixed retention for lifetime.

Type and severity of malocclusion and choice of retainer.In age Group 1 (6-12y.)was established thatthe removable retainers were more often used, which corresponded to the higher percentage of patients, treated with removable orthodontic appliances in this age group. With increasing the age and complication of the pathology, the greater safety of the retention can be achieved by combining the retention methods (FR + VR) or by making them more stable and reliable- double fixed retainer. This is connected with the changes, which occur in the supporting tissues - more frequent periodontal diseases in adult patients and reduced compensatory abilities.

The evaluation of extraction cases in the current study showed a higher usage of FR from premolar to premolar (44). This was done to reduce the risk of opening the extraction gaps. In order to maintain a good result and to ensure stability after closing diastemas/spacing [13] as well as in cases of severe crowding $[15,16]$, a FR was also the preferred retention. According to Little,the better choice for prevention of relapse in cases needed long-term retention, is the fixed retainer $[15,16]$.

When treating deep bite patients the better retention is FR in the lower jaw in a combination withTrainer for night use. This can be recommended because the Trainer has an influence over the muscles - make a balance between the inner (tongue) and outer muscle belt (cheeks and lips), levels the occlusal plane and respectively raises the bite so a long-term stable result can achieved. In cases with impacted wisdom teeth and contraindications for their extraction and / or non-cooperative patients, there is a higher risk of relapse and crowding in lower front segment. So an enhanced retention of FR and VRis recommended in combination with relieving stripping over a certain period of time. Although, according to some studies the extraction of third molars to prevent crowding in frontal area or the relapse after orthodontic treatment is not justified [17].

Some studies show that additional methods like approximal stripping [18] and circumferential supracrestalfibertomy $[19,20]$, parallel with the use of removable retainer, are more effective, especially for relapse prevention in cases with rotated teeth. Circumferential supracrestalfibertomy have no harm effect on dental and periodontal health [19].

In open bite cases, the retention phase was done with VR and attachments for intermaxillary traction.
Fixed retainers were indicated in cases with reduced periodontal support. The adult patients have greater requirements for the retention appliances - not to disturb the aesthetics, speech, nutrition and not to violate their social life. Hence the need for aesthetic and casual retention works. This determines the FR as the preferred method for retention following an orthodontic treatment.

Periodontal status and selection of retainer.All patients in that group have completed interdisciplinary orthodonticperiodontal treatment. Diagnosis of the patients was made byspecialist - periodontologist. After the completion of initial and active periodontal therapy, and the supportingperiodontal therapy was started, the orthodontic treatment could be started. The enhanced retention (FR + VR, DFR) in the group aimed to reduce the chewing pressure on teeth with reduced periodontal tissues. In these cases the normal chewing pressure became pathologic, as it distributes on lower root surface. This requires splinting the teeth and hence increase the total surface, which reduces the stress over the paradental tissues and gives the opportunity to stabilize hard dental tissues.

Compared to periodontal health it was found that along with increasing the severity of pathology the need for enhancing the retention is alsoincreased (combination of FR + VR, DFR).

According to Watted's study of 60 patients, separated in 3 groups (two of which are with fixed retainer and one with removable retainer) the dental mobility reduces with the number of teeth, fixed in the retainer [21]. This conclusion was supported by a study of Stormann, which recruited 103 patients [22]. It proves that canine to canine fixed retainer gives a good level of stability compared to canine and canine retainer where the incisors are free. In a literature review of Bearnit is seen that the more rigid and larger diameter wire would increase the force necessary to obtain a permanent deformation and therefore reduces the risk of relapse [23].

The advantages of fixed mandibular retainer are: reduced cooperativeness of the patient, better aesthetics, reduced risk of relapse of the lower frontal segment with longer wear $[12,23]$.It allows a physiological displacement of the teeth while maintenance of their position [9].Fixed retainers are also indicated in cases with reduced periodontal support, and for retention after treatment of diastema [24]. Nevertheless, Lumsden and Artunobserved more than 47\% failure rate of fixed retainer [22], especially in the upper incisors and in deep bite cases [25, 26]. Compared to removable retainers it can be added and greater accumulation of plaque and tartar [27]. Therefore, they require greater and sustained support. Now is recommended the use of thicker multi-threading wire.

Type of orthodontic appliances and choice of retainer.According to this study when treated with removable appliance it later remains as a retainer. For example, a plate used for treatment in childhood (group 1), later can be used as a retainer appliance after completion of the active orthodontic treatment. This is in order not to suppress the three-dimensional growth of the jaws. Lingual 


\section{International Journal of Science and Research (IJSR) \\ ISSN (Online): 2319-7064}

Index Copernicus Value (2015): 78.96 | Impact Factor (2015): 6.391

plate has no reliable components for vertical control, making it ineffective retainer after treatment with fixed appliances. During treatment with Trainer, it remains as a support appliance, because its function is to eliminate bad habits that lead to various distortions, to balance the muscles and thus to provide long-term stable results; in treatment with aligners, the later remains for retention in cases with good cooperativeness of the patient (Fig. 3); when treated with fixed appliances the most commonly used retainer is FR (80.4\%), and in cases with treatment with EOA - a combination of fixed and vacuum retainer (54.9\%) was used.
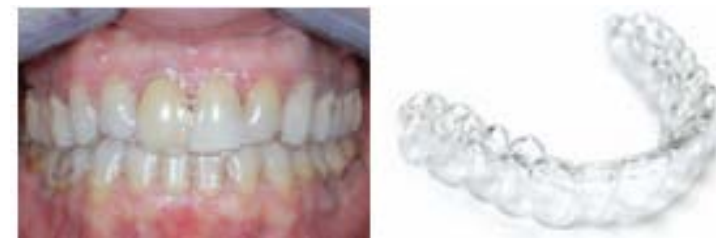

Figure 3: Patient treated with Dent@lign aligners. The last ones were used for retention.

Treated jaw and choice of retainer. The study showed that in upper jaw the most commonly used retainer was the FR (3-3), followed by FR (2-2) and VR. In lower jaw, FR (3-3) was also the most preferable method for retention, followed by a combination of FR and VR, and at last - FR (4-4) (in extraction cases). In the presence of wisdom teeth, especially in lower jaw, the risk of relapse is higher, as it occurs mainly in the frontal segment, because of the gracile roots of the lower incisors. In addition, throughout life teeth tend to medialize - this can lead to relapse after treatment of crowding in the upper and lower jaw.

Cooperativeness of the patient and choice of retainer.Patients showing good compliance during treatment (respecting appointments of regular visits, supporting bracketsystem, respecting the instructions given to carry / storage / cleaning appliances) are provided with removable retention appliances (vacuum retainers) in the majority of cases. In non-cooperative patientsfixed retainers are made.

Study ofAttack et al., which recruited 58 patients divided into two groups-one with removable retainer type Hawley, and the other with fixed retainer, shows that the incidence of relapse in the lower frontal segment in the group with removable retainer is significantly greater. However, relapse was observed, and in the group with fixed retainer. This is explained by the need for better adherence by the patient in the use of removable appliances unlike fixed. The irregular wear of the appliances leads to a higher risk of teeth displacement, relapse in the lower frontal segment or broken fixed retainer [12].

\section{Conclusion}

Retention is an important part of the orthodontic treatment. The choice of retainer depends on the patient's age, type and severity of malocclusion, used treatment appliances, periodontal condition and cooperativeness of the patient. Orthodontic retainers counteract the natural tendency of teeth to return to their original position under the influence of periodontal, occlusal, soft tissue forces, as well as continuing dental-facial growth. Type of retainer highly determines the reliability of the retention and stability of the result.

\section{References}

[1] Joondelph DR, Riedel RA. Retention and Relapse. In: Graber TM, Vanarsdall RL, editors. Orthodontics Current Principles and Techniques. 2nd ed. St. Louis: Mosby; 1994:908-950.

[2] Bearn DR. Bonded orthodontic retainers: A review. Am J OrthodDentofacOrthop. 1995;108:207-213.

[3] Reiten K. Clinical and histologic observations on tooth movement during and after orthodontic treatment. American Journal of Orthodontics 1967; 53:721-745.

[4] Edwards JG. A study of the periodontium during orthodontic rotation of teeth. American Journal of Orthodontics 1968; 54: 441-461.

[5] Clark JD, Kerr WJ, Davis MH.Cases-clinical audit; scenarios for evaluation and study.British Dental Journal 1997; 183; 108:111.

[6] Joondelph DR, Riedel RA. Retention and Relapse In: Graber TM, Vanarsdall RL, eds. Orthodontics Current Principles and Techniques. $2^{\text {nd }}$ ed. St.Louis: MosbyYear Book, 1994: 908-950.

[7] Sharpe W, Reed B, Subtenly JD, Polson A. Orthodontic relapse, apical root resorption, and crestal alveolar bone levels. AJODO March 1987; 91(3): 252-258.

[8] Kneirim R. Invisable lower cuspid to cuspid retainer. The Angle Orthodontist 1973; 43(2): 218- 20.

[9] Zachrisson BU 1997 Clinical experience with directbonded orthodontic retainers. American Journal of orthodontics 71: 440-48.

[10]Zachrisson, B. U. Third - Generation Mandibular Bonded Lingual 3-3 Retainer. - JCO, 1995, 29 (1): 3948.

[11] Zachrisson B. The bonded lingual retainer and multiple spacing of anterior teeth. Sweed Dent Suppl 1982;15:247-55.

[12] Attack N., N. Harradine, J.R.Sandy, Which way forward? Fixed or Removable lower Retainers. Angle Orthodontist 2007, 77(6): 954-9.

[13] Andren A, Asplund J, Azarmidohkt E, Svensson R, Varde P, Mahlin B. A clinical evaluation of long term wires. Swed Dent J 1998; 22: 123-131.

[14] Proffit WR, Fields HW, Sarver DM. Contemporary Orthod. (4 $\left.{ }^{\text {thed. }}\right)$, Chap. 17, St Louis, Mosby Elsevier, 2007.

[15] Little R M, Reidel R A, Artun J 1988 An evaluation of changes in mandibular anterior alignment from 10 to 20 years postretention. American Journal of Orthodontics and Dentofacial Orthopedics 93: 423-428.

[16] Little R M, Wallen T R, Reidel R A, Stability and relapse of mandibular anterior alignment - first premolar extraction cases treated by traditional Edgewise orthodontics. American Journal of Orthodontics 1981; 80: 349- 365.

[17]ZawawiK., M. Melis. The role of mandibular third molars onlower anterior teeth crowding and relapse afterorthodontic treatment: asystematic review. Scientific World Journal2014, Article ID 615429, 6 pages. 


\section{International Journal of Science and Research (IJSR) \\ ISSN (Online): 2319-7064}

Index Copernicus Value (2015): 78.96 | Impact Factor (2015): 6.391

[18] BoeseLR.Fiberotomy and reproximation without loer retention, nine years in retrospect: Part I. Angle Orthod. 1980; 50: 88-97.

[19] Taner T, Hayder B, Kavuklu I, Korkmaz A. Short-term effects of fibertomy on relapse of anterior crowding. American Journal of Orthodontics and Dentofacial Orthopedics 2000; 118: 617- 623.

[20] John GE. A long-term prospective evaluation of the circumferential supracrestalfiberotomy in alleviating orthodontic relapse. American Journal of Dentofacial Orthopedics 1988; 93(5): 380- 387.

[21] Watted, N. M. Wieber et al, Comparison of incisor mobility after insertion of canine-to-canine lingual retainers bonded to two or to six teeth. A. clinical study. Journal of Orofacial Orthopedics 2001; 62(5): 387-396.

[22] Stormann, I, E. Ulrike, A prospective randomized study of different retainer types. Journal of Orofacial Orthopedics 2002; 63: 42-50.

[23] Sadowsky, C, Schneider BJ, BeGole EA, Tahir E. Long-term stability after orthoonti treatment: nonextraction with prolonged retention. Am J OrthodDentofacialOrtop. 1994; 106: 243-249.

[24]Bearn DR. Bonded orthodontic retainers: a review. American Journal of Orthodontics and Dentofacial Orthopedics 1995; 108: 207-213.

[25] Lumsden KW, Saidler G, McColl JH. Breackage incidence with direct-bonded lingual retainers. British Journal of Orthodontics 1999; 26: 191-4.

[26] Artun J, Ubrye KS. The effect of orthodontic treatment on periodontal bone support in patients with advanced loss of marginal periodontium. American Journal of Orthodontics and Dentofacial Orthopedics 1988; 93: 143-148.

[27]Heier EE, De Smit AA, Wijgaerts IA, Adriaens PA. Periodontal implications of bonded versus removable retainers. American Journal of Orthodontics and Dentofacial Orthopedics 1997; 112: 607-616.

\section{Author Profile}

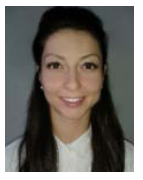

NikoletaRumenovaIvanova, DMD, graduated the Faculty of Dental Medicine, Medical University of Sofia in 2014. She has an interests in functional and interceptive orthodontics. DrIvanova is a member of BSCLO.

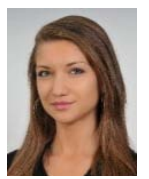

Nora GeorgievaGogusheva, DMD, graduated Dental Medicine in 2016 at Faculty of Dental Medicine, Medical University of Sofia, Bulgaria. Her interests are focused in Orthodontics and Esthetic Dentistry.

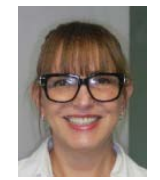

MiroslavaMiletiDinkova, $\mathrm{DMD}, \mathrm{PhD}$ entered the field of dentistry in 1978 and specialized in Orthodontics, Pediatric Dentistry, Health Management and General Dentistry in 1987, 1993 and 2005, respectively at Faculty of Dental Medicine, Medical University of Sofia, Bulgaria. In 2014 she received her $\mathrm{PhD}$ degree in Orthodontics. Since 1992 she is Assistant Professor and since 2015 - Associate Proffesorat the Department of Orthodontics - Medical University of Sofia, Bulgaria. Her main interests are in adult orthodontics, interdisciplinary treatment approaches, lingual orthodontics and digital technologies in orthodontics. Dr. Dinkova is a member of WFO, EOS, SIDO, BaSS, BOS and President of BSCLO. 